\title{
Rhein sensitizes human colorectal cancer cells to EGFR inhibitors by inhibiting STAT3 pathway
}

This article was published in the following Dove Press journal: OncoTargets and Therapy

\author{
Yan Zhuang ${ }^{1,2, *}$ \\ Ying $\mathrm{Bai}^{1,2, *}$ \\ Yan $\mathrm{Hu}^{3, *}$ \\ Yueqin Guo ${ }^{1,2}$ \\ Lingyuan $\mathrm{Xu}^{1,2}$ \\ Wanle $\mathrm{Hu}^{4}$ \\ Lehe Yang ${ }^{1,2}$ \\ Chengguang Zhao ${ }^{1,2}$ \\ Xiaokun $\mathrm{Li}^{1,2}$ \\ Haiyang Zhao ${ }^{1,2}$ \\ 'The Institute of Life Sciences, Wenzhou \\ University, Wenzhou, Zhejiang 325035, \\ People's Republic of China; ${ }^{2}$ School of \\ Pharmaceutical Sciences, Wenzhou \\ Medical University, Wenzhou, Zhejiang \\ 325035, People's Republic of China; \\ ${ }^{3}$ Department of Pharmacy, Taizhou Enze \\ Medical Center (Group), Taizhou \\ Hospital of Zhejiang Province, Taizhou, \\ Zhejiang 317000, People's Republic of \\ China; ${ }^{4}$ Department of Coloproctology, \\ The Second Affiliated Hospital and Yuying \\ Children's Hospital, Wenzhou Medical \\ University, Wenzhou, Zhejiang 325000, \\ People's Republic of China
}

*These authors contributed equally to this work

Correspondence: Xiaokun Li

School of Pharmaceutical Sciences,

Wenzhou Medical University, University

Town, Wenzhou, Zhejiang 325035,

People's Republic of China

Email profxiaokunli@I63.com

Haiyang Zhao

The Institute of Life Sciences, Wenzhou University, University Town, Wenzhou,

Zhejiang 325035, People's Republic of

China

Tel +865 7786699057

Fax +865778 6699057

Email haiyangwzu@I63.com
Background: Activation of epidermal growth factor receptor (EGFR) has been reported in a variety of cancer types, including colorectal cancer (CRC), and represents a potential chemotherapeutic drug target. EGFR tyrosine kinase inhibitors (EGFR-TKIs) have been increasingly applied in the clinical treatment of CRC, but development of drug resistance during the treatment has greatly limited their application. Signal transducer and activator of transcription 3 (STAT3) and its mediated signal transduction pathway play an important role in the occurrence, development and metastasis of CRC, and are related to the development of EGFR-TKI resistance in CRC.

Methods: Cell viability, colony formation and cellular morphology were examined to evaluate the potent antiproliferative effect of the STAT3 inhibitor napabucasin, LY5 and rhein on the human CRC cell lines HCT116, SW620, RKO and DLD-1. Flow cytometrybased analysis was employed to determine whether rhein can affect the cell cycle and apoptosis. The expression level of phosphorylated STAT3 (P-STAT3), and cell cycle- and apoptosis-related proteins BCL2, CDC2 BAX, Cyclin D1 and Cyclin B1 were detected by Western blot analysis.

Results: This study revealed that rhein can significantly reduce cell viability and stimulate apoptosis in human CRC cells in a dose-dependent manner. In addition, rhein induced cell cycle arrest at the G2/M phase in CRC cells and dose-dependently inhibited the expression of cell cycle-related proteins. Additionally, it was found that napabucasin, LY5 and rhein considerably sensitized cells to the EGFR-TKI erlotinib, thus suppressing CRC cell proliferation. Rhein also inhibited the phosphorylation of its downstream target STAT3. Inhibition of STAT3 and EGFR phosphorylation was also observed after treatment with a combination of rhein and EGFR inhibitors.

Conclusion: This study confirmed the synergistic effect of STAT3 inhibitor and EGFR inhibitor in CRC cell lines. Additionally, we found that rhein sensitizes human CRC cells to EGFR-TKIs by inhibiting STAT3 pathway. When combined with EGFR-TKIs, rhein may be a novel STAT3 inhibitor in CRC.

Keywords: rhein, STAT3, colorectal cancer, EGFR, tyrosine kinase inhibitor

\section{Introduction}

Colorectal cancer (CRC) is one of the most commonly diagnosed and lethal cancers worldwide. The prognosis of CRC patients depends on the cancer stage at diagnosis. ${ }^{1}$ Detection of $\mathrm{CRC}$ at an early stage may lead to a $90 \% 5$ year survival rate, compared to $12 \%$ when distant metastasis has occurred. ${ }^{2,3}$ The epidermal growth factor receptor (EGFR) signaling pathway plays an important role in the proliferation, angiogenesis, invasion and metastasis of CRC cells. ${ }^{4}$ EGFR tyrosine kinase inhibitors (EGFR-TKIs) have been increasingly applied in the clinical 
treatment of CRC. ${ }^{5}$ However, development of resistance to EGFR-TKI drugs during treatment has greatly limited their application. In patients with $\mathrm{CRC}$, secondary drug resistance may occur after 10-16 months of treatment, even when early treatment is effective. ${ }^{6,7}$ Development of drug resistance has become the bottleneck of EGFRTKIs therapy, which increases the difficulty of clinical treatment of CRC. ${ }^{8,9}$ Considerable basic and clinical research is still needed to study the mechanism of drug resistance.

Signal transducer and activator of transcription 3 (STAT3), an important member of the STAT protein family, which are recognized as oncogenes for their role in the malignant transformation of cells and tumorigenesis. $^{10,11}$ Recent studies have shown that STAT3 and its mediated signaling pathway play an important role in EGFR-TKI resistance in CRC cells. ${ }^{12}$ The feedback activation of STAT3 is a new mechanism for the development of drug resistance to EGFR inhibitors in CRC cells. ${ }^{13-15}$ Thus, to overcome drug resistance and better treat $\mathrm{CRC}$, new strategies need to be developed to improve the effectiveness of EGFR inhibitors, especially by blocking the STAT3 pathway. ${ }^{16}$ We found that the STAT3 inhibitors combined with EGFR inhibitors, had synergistic anti-proliferative and pro-apoptotic effects in vitro and in vivo. ${ }^{12,17}$

Rhein is a lipophilic anthraquinone widely found in medicinal plants. Emerging evidence suggests that rhein has remarkable antitumor effects, supporting its potential use as an antitumor agent. ${ }^{12,18}$ Furthermore, we also found that rhein sensitizes human CRC cells to EGFR inhibitors by inhibiting STAT3 pathway. Thus, when combined with EGFR-TKI, rhein may be a novel STAT3 inhibitor in CRC cells.

\section{Materials and methods}

\section{Cell lines and antibody}

Rhein was purchased from TagerMol (Shanghai, China) and its purity is $98.7 \%$. DMSO was used as a solvent to dissolve all drugs. Human colon cancer cell lines (HCT116, SW620, RKO and DLD-1) were obtained from cell resources center of the Shanghai Institutes for Biological Sciences (Chinese Academy of Sciences, Shanghai, China). HCT116 and RKO cells were grown in McCoy's 5A medium (Gibco/BRL; Thermo Fisher Scientific, Waltham, MA, USA) supplemented with $10 \%$ fetal bovine serum (FBS) (Gibco; Life Technologies,
Carlsbad, California, USA) and 1\% of antibiotic solution (100 units $/ \mathrm{mL}$ penicillin and $100 \mu \mathrm{g} / \mathrm{mL}$ streptomycin) in a humidified atmosphere of $5 \% \mathrm{CO}_{2}$ at $37^{\circ} \mathrm{C}$. SW620 and DLD-1 cells were cultured in Dulbecco's Modified Eagle's Medium (DMEM; Thermo Fisher Scientific; Suzhou, China) with 10\% FBS (Gibco; Life Technologies, Carlsbad, California, USA), 1\% of antibiotic solution and incubated at $37^{\circ} \mathrm{C}$ with $5 \% \mathrm{CO}_{2}$. The antibodies against P-STAT3, STAT3, P-EGFR, EGFR, CDC2, CyclinB1, CyclinD1, BCL-2, BAX, GAPDH, horseradish peroxidase (HRP)-conjugated donkey antirabbit $\mathrm{IgG}$ and $\mathrm{HRP}$-conjugated goat anti-mouse $\mathrm{IgG}$ were purchased from Santa Cruz Biotechnology Inc. (Dallas, TX, USA).

\section{MTT assay}

Cells were seeded into wells of a 96-well plate at $5 \times 10^{3}$ cells per well in $100 \mu \mathrm{L}$ of the corresponding medium. Rhein was dissolved in DMSO to a final concentration of $0,25,50,75,100 \mu \mathrm{M}$. CRC cell lines were incubated with rhein for $48 \mathrm{~h}$ or $72 \mathrm{~h}$ hours. Subsequently, cells were treated with a fresh solution of MTT $(5 \mathrm{mg} / \mathrm{mL})$ for $4 \mathrm{~h}$ at $37^{\circ} \mathrm{CC}$. The purple formazan crystals were finally solubilized with DMSO solution, and absorbance was recorded using a multi-well plate reader at $490 \mathrm{~nm}$.

\section{Western blot analysis}

Cells were lysed in protein lysis buffer and centrifuged at $12,000 \mathrm{rpm}$ for $10 \mathrm{mins}$ at $4^{\circ} \mathrm{C}$ to remove nuclei and cell debris. The cell extract concentrations were determined with the Bradford protein assay kit (Bio-Rad Laboratories Inc., Hercules, CA, USA). After addition of sample loading buffer, proteins were separated by electrophoresis and the proteins were then electroblotted to poly-vinylidene difluoride transfer membranes. The blots were blocked for $2 \mathrm{hrs}$ with fresh 5\% non-fat milk in tris-buffered saline with Tween 20 (TBST) buffer at room temperature, followed by incubation with primary antibody in TBST overnight at $4{ }^{\circ} \mathrm{C}$. After washing three times with TBST, the blots were incubated with horseradish peroxidase-conjugated secondary antibodies for $1 \mathrm{hr}$. After three washes with TBST, the antibody staining was visualized using the ECL kit (BioRad Laboratories Inc. Hercules, CA, USA). Then, the images were analyzed using the Image $\mathrm{J}$ computer software (National Institute of Health, Bethesda, MD, USA). 


\section{Cell cycle analysis}

Cells were seed into 6-well plate for $24 \mathrm{hrs,} \mathrm{and} \mathrm{then}$ treated with DMSO or different concentrations of rhein and erlotinib for $24 \mathrm{hrs}$. Cells were then labeled with propidium iodide (PI), and the cell cycle was analyzed by flow cytometry analysis (BD Biosciences, San Jose, CA, USA).

\section{Analysis of cell apoptosis}

Cells $\left(3 \times 10^{5}\right.$ cells/well) were seeded in 6-well plate and incubated overnight and then treated with rhein and erlotinib for $48 \mathrm{~h}$. After treatment, the cells were harvested with trypsin and then washed with cold PBS twice. The cells were stained with Annexin V for 10 min under dark conditions and then with PI for 5 min. Annexin V-binding buffer was then added to the mixture before fluorescence was measured on a FACSCalibur (BD Biosciences; Baltimore, MD, USA). Data were analyzed using Flowjo software.

\section{Scratch wound model}

Cells $\left(5 \times 10^{5}\right.$ cells/well $)$ were seeded in 6 -well plate and allowed to adhere overnight. At $80-90 \%$ confluence, a "reference line" was scratched at the bottom of the plate by using a sterile $10 \mu \mathrm{L}$ pipette tip. After being washed with phosphate buffer saline (PBS) thrice, cells were further incubated with inhibitors or a vehicle (DMSO) in a calcium-free medium to examine cell migration in the absence of cell growth. Photomicrographs of cells migrating across the reference line were taken in different fields with a Canon digital camera (Tokyo, Japan) after treatment at 0 and $48 \mathrm{~h}$, respectively. The rate of mobility was quantified by the migrated distance of cells moved from the reference lines toward the center, compared with that of the control. All experiments were repeated 3 times.

\section{Clonogenic assay}

A total of 500 cells per well were seeded into a 6-well plate with $2 \mathrm{~mL}$ medium overnight. The cells were then pretreated with ihibitors or DMSO for 8-12 h. After treatment, the cells were washed with PBS twice and then transferred into a fresh medium to allow cells to grow for 7 days. Colonies were washed with PBS and then fixed with $4 \%$ methanol for $15 \mathrm{~min}$ at room temperature. The cells were washed with PBS twice and then stained with $1 \%$ crystal violet ( $25 \%$ methanol) for $10 \mathrm{~min}$ at room temperature. Each experiment was conducted for 3 independent experiments.

\section{Statistical analyses}

Data are represented as mean \pm standard error of the mean of 3 independent experiments. Student's $t$-test was performed to determine the statistical significance between 2 groups by using GraphPad Prism 7.0 (GraphPad Software, Inc., San Diego CA, USA). $P<0.05$ was considered statistically significant.

\section{Results}

\section{Synergistic effect of STAT3 inhibitors and erlotinib on CRC cells proliferation}

The synergistic effect of the STAT3 inhibitor LY5 and the EGFR inhibitor erlotinib on human CRC cells was investigated in the HCT116 and DLD-1 CRC cell lines treated with STAT3 inhibitor LY5 and erlotinib, separately or in combination, for 48 or $72 \mathrm{~h}$, by evaluating cell viability using the MTT assay. The inhibitory effect of the combination of LY5 with erlotinib on CRC cells was statistically significant and more pronounced than that of LY5 or erlotinib alone, with almost all of combination index CI $<0.5$ (Figure 1A and B). Also, as anticipated, in HCT116, DLD-1 and RKO cells, the cell scratch test demonstrated that the combination of LY5 or another STAT3 inhibitor napabucasin and erlotinib synergistically inhibited cell migration more significantly than that either inhibitor alone (Figure 2A-C). Moreover, napabucasin combined with erlotinib had a more obvious inhibitory effect on migration factor MMP-2 than napabucasin or erlotinib alone in RKO cells (Figure 2D).Together, these results showed that the combination of STAT3 inhibitor and EGFR inhibitor exerted a significant synergistic cell growth inhibitory on human CRC cells.

\section{Rhein exerts antitumor effect and sensitizes EGFR inhibitors by inhibiting STAT3}

The effect of rhein on cell viability was determined in the two human CRC cell lines HCT116 and SW620, using the MTT assay. The results of the MTT assay revealed that rhein reduced the viability of HCT116 and SW620 cells with IC50 values of 15.4 and $17.2 \mu \mathrm{M}$, respectively (Figure 3A). Furthermore, the Western blot analysis revealed that rhein significantly reduced the expression of P-STAT3 and activated P-EGFR in HCT116 cells. Notably, rhein significantly inhibited STAT3 phosphorylation and activated P-EGFR in 
A

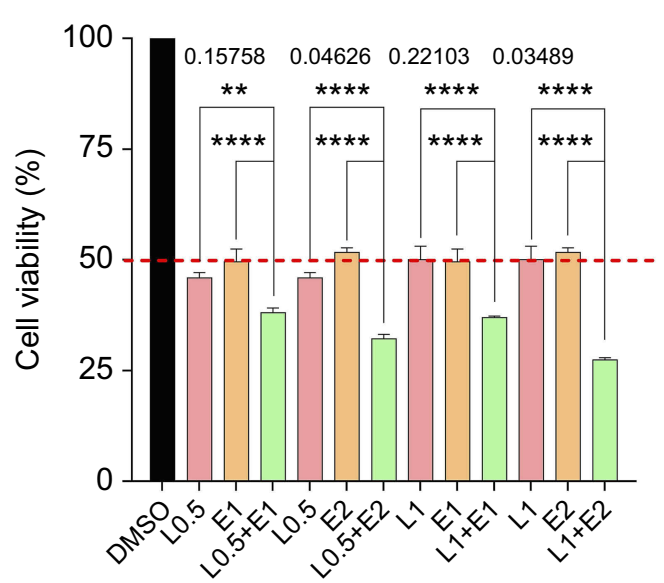

B

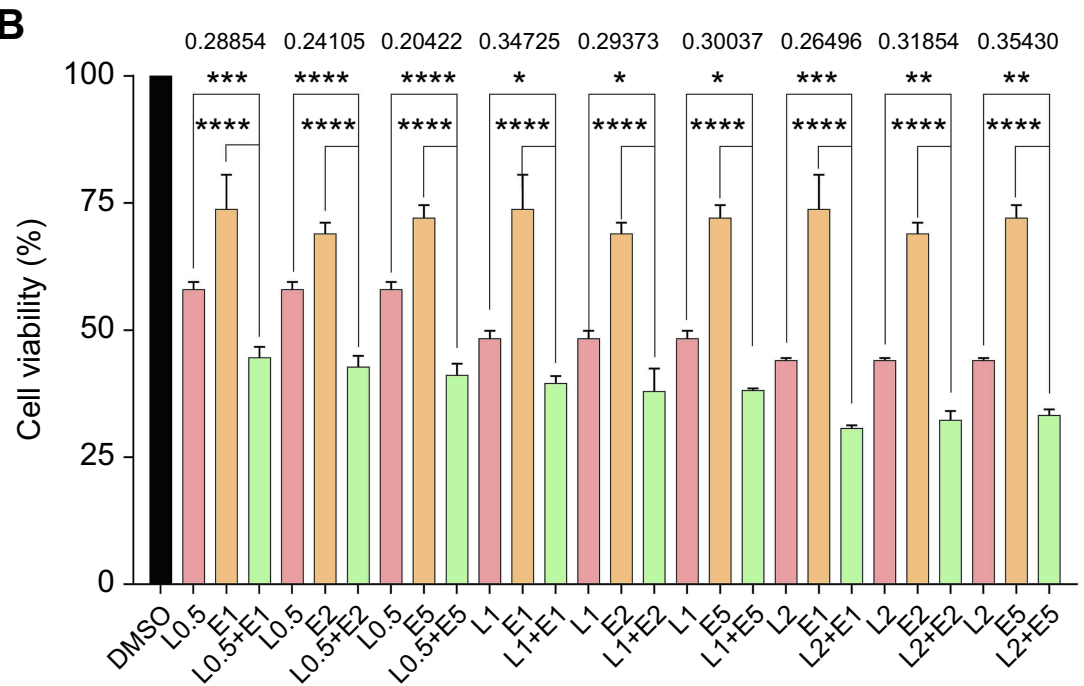

Figure I The combined effect of LY5 and Erlotinib on CRC cell proliferation measured by the MTT assay. (A) HCTI I6 cells were treated, in combination or separately with the STAT3 small molecule inhibitor LY5 and/or EGFR small molecule inhibitor erlotinib at different concentrations for $72 \mathrm{~h}$. (B) DLD-I cells were treated in combination or separately with LY5 and/or erlotinib at different concentrations for $48 \mathrm{~h}$. $(* P<0.05, * * P<0.0 \mathrm{I}, * * * P<0.00 \mathrm{I}, * * * * P<0.000 \mathrm{I})$.

Abbreviations: DMSO, dimethyl sulfoxide; L, LY5; E, erlotinib; CRC, colorectal cancer; EGFR, epidermal growth factor receptor.

a time-dependent manner (Figure 3B-D). Then, we evaluated the effect of rhein on the activation of JAK2 in HCT116 cells. However, rhein did not reduce the protein levels of phosphorylated JAK2. This result suggested that rhein inhibits STAT3 phosphorylation independent of the upstream kinases JAK2 (Figure 3E). This finding suggested that the effects of rhein are highly selective for the STAT3. Furthermore, rhein can inhibit IL6 induced activation of STAT3 pathway (Figure $3 \mathrm{~F}-\mathrm{G}$ ). Moreover, treatment of CRC cells with the combination of erlotinib and rhein significantly attenuated cell viability compared to cells treated with either inhibitor alone (Figure $3 \mathrm{H}$ ). In summary, in combination with erlotinib, rhein exerted a synergistic and stronger inhibitory effect through inhibiting STAT3 pathway on CRC cells.

\section{Erlotinib and rhein synergistically induce apoptosis in human CRC cells}

Compared with single-agent treatment, the combination of erlotinib and rhein enhanced the inhibition of cell viability in all tested cell lines. We sought to investigate whether the combination of erlotinib and rhein could also act synergistically to induce cell apoptosis. To this end, we used flow cytometry to examine whether the combination of erlotinib and rhein induced cell apoptosis in CRC cell lines. HCT116 and SW620 cells were treated with erlotinib $(10 \mu \mathrm{M})$ and/or rhein $(100 \mu \mathrm{M})$ for $24 \mathrm{~h}$ and then stained with Annexin V and propidium iodide (PI) for cell apoptosis analysis. Data analysis showed that the combination of the two drugs was more effective at inducing 
A
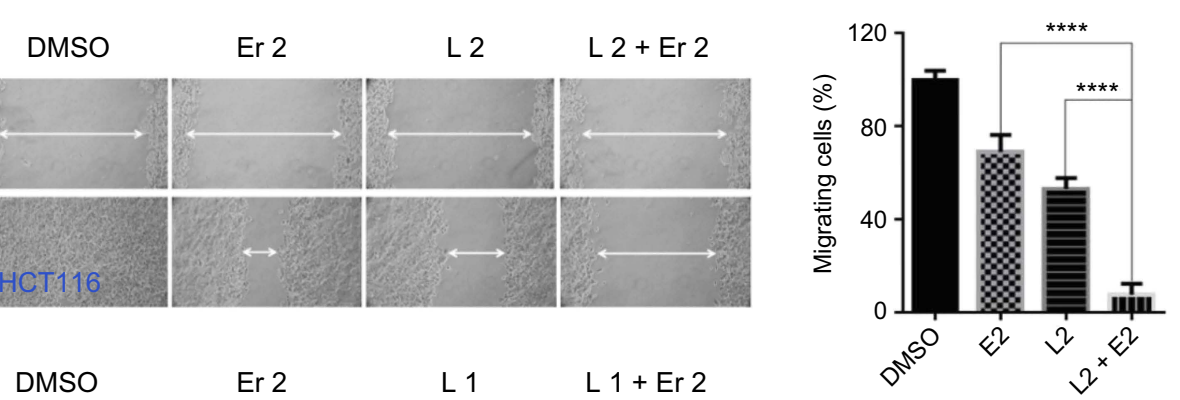

B

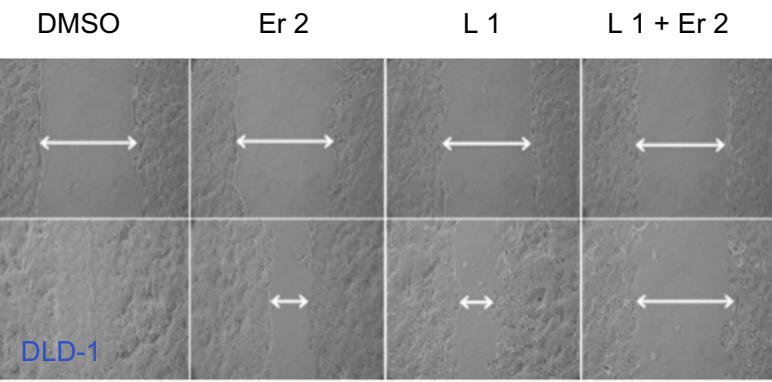

C
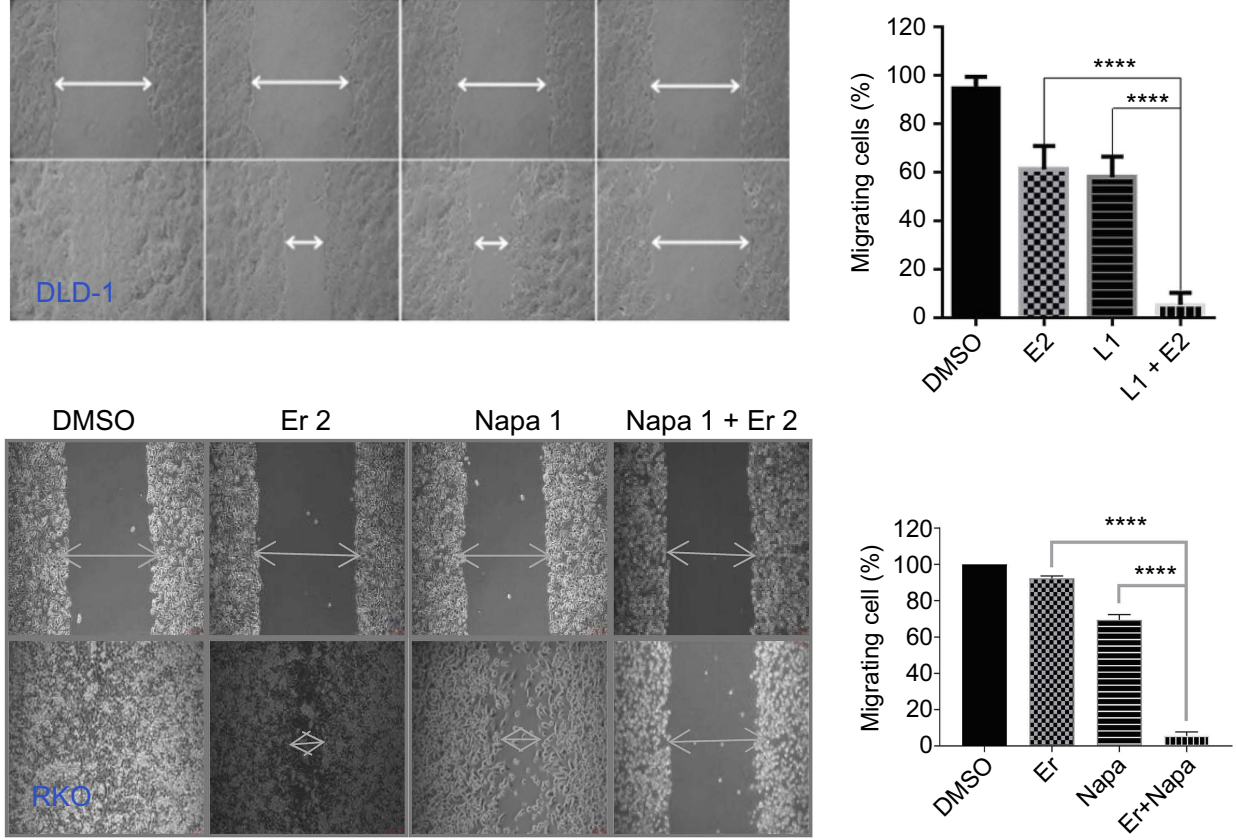

D
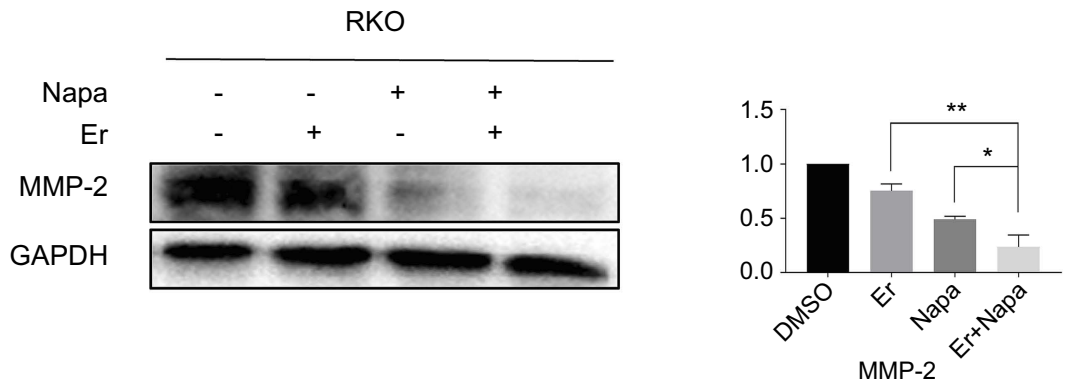

Figure 2 The combined effect of STAT3 inhibitors and erlotinib on CRC cell migration by wound healing assay. (A) HCTII6 cells were treated, in combination or separately, with EGFR small molecule inhibitor erlotinib $(E r, 2 \mu M)$ and/or STAT3 small molecule inhibitor L (LY5, $2 \mu M)$ and allowed to migrate into the scratched area for $24 \mathrm{~h}$. (B) DLD-I cells were treated, in combination or separately, with $\operatorname{Er}(2 \mu \mathrm{M})$ and/or $L(I \mu M)$ and allowed to migrate into the scratched area for 24 h. (C) RKO cells were treated, in combination or separately, with $\operatorname{Er}(2 \mu \mathrm{M})$ and/or napabucasin $(1 \mu \mathrm{M})$ and allowed to migrate into the scratched area for $24 \mathrm{~h}$. Wound healing assay for migration was conducted by scratching the cells with a yellow tip when cells grew into a monolayer. (D) RKO cells were treated, in combination or separately, with Er ( $2 \mu \mathrm{M})$ and/or napabucasin $(\mathrm{I} \mu \mathrm{M})$ to verify the effect on MMP-2 protein. Data were obtained from 3 independent experiments. $(* P<0.05$, $* * P<0.0 \mathrm{I}$, $* * * * P<0.000 \mathrm{I})$.

Abbreviations: CRC, colorectal cancer; EGFR, epidermal growth factor receptor.

apoptosis that either of the two drugs individually. The combination treatment resulted in a significant increase in early and late apoptotic cells, as shown in Figure 4A. The experimental results indicated that the rate of apoptosis was significantly higher in cells treated with the combination of the two drugs, further indicated that the combination treatment of erlotinib and rhein had a synergistic anti-CRC effect (Figure 4B). 
A

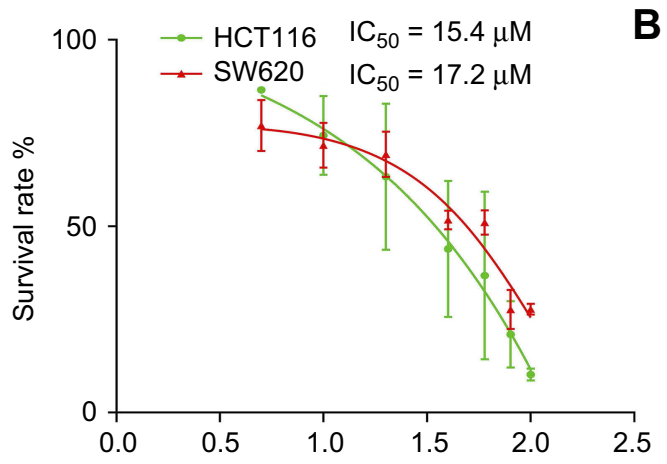

C

E
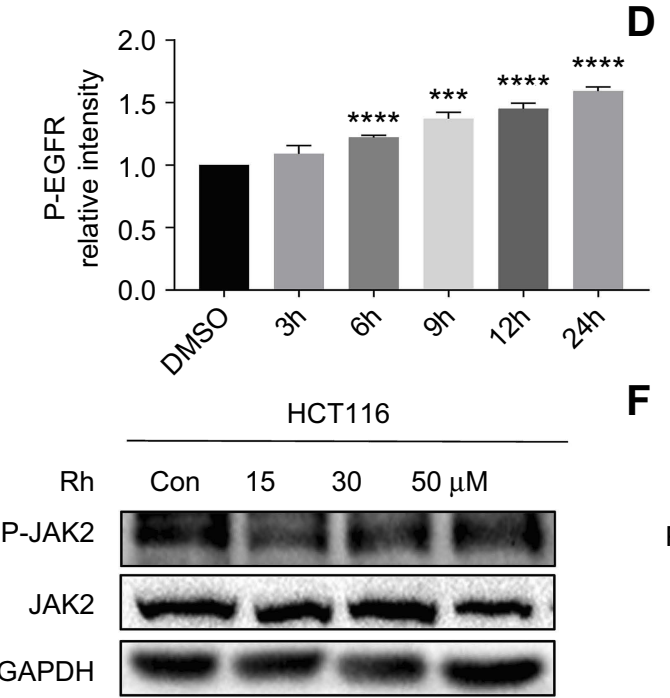

B
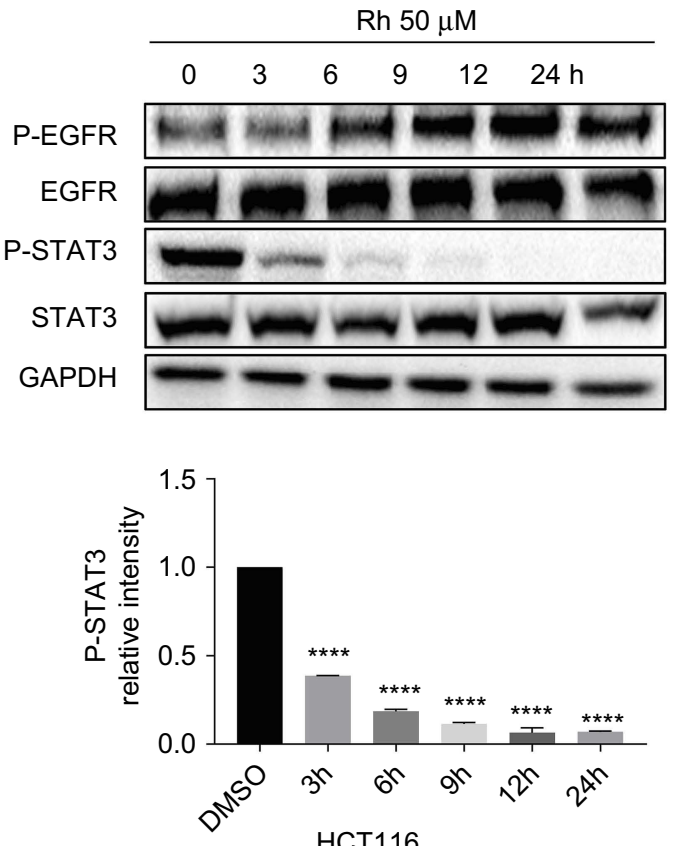

$\mathbf{F}$

IL-6

$\mathrm{Rh}$

\begin{tabular}{ccccl}
\multicolumn{6}{c}{ HCT116 } \\
\hline- & + & + & + & + \\
- & - & 15 & 30 & $50 \mu \mathrm{M}$
\end{tabular}

P-STAT3

STAT3

GAPDH

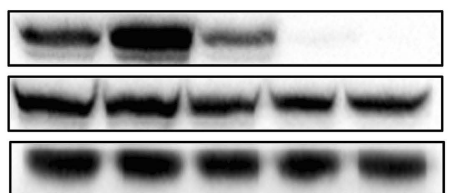

G

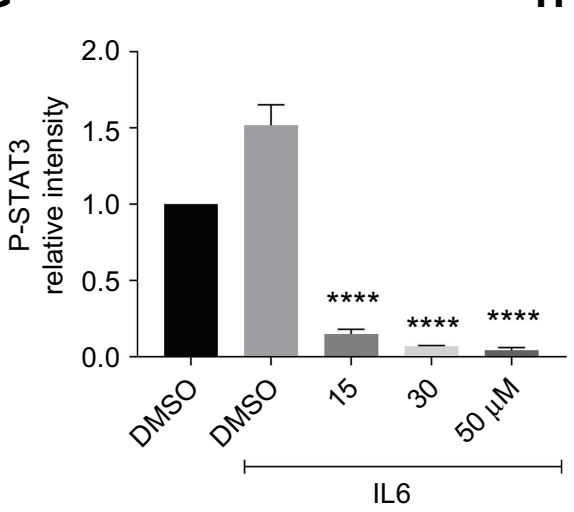

H

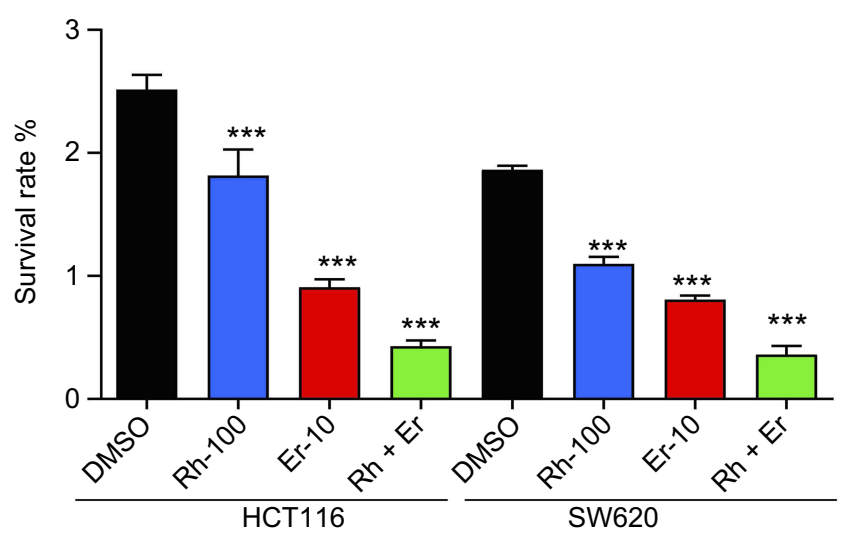

Figure 3 Rhein sensitizes human CRC cells to EGFR inhibitors by inhibiting STAT3 pathway. (A) HCTII6 and SW620 cells were seeded in a 96 -well plate at a density of 5,000 cells per well and then cultured for $24 \mathrm{~h}$. The cells were treated with rhein at the indicated concentrations. After a $48 \mathrm{~h}$ treatment, cell proliferation in each group was measured by the MTT assay, the IC50 is indicated. (B) HCTII6 cells were treated with rhein $(50 \mu \mathrm{M})$ for different periods of time $(0,3,6,9,12$ and $24 \mathrm{~h})$. Total protein was extracted, and the expression levels of P-EGFR, EGFR, P-STAT3, STAT3 and GAPDH proteins were measured by Western blot analysis. (C) Western blot analysis results from P-EGFR were calculated. (D) Western blot analysis results from P-STAT3 were calculated. (E) HCTII6 cells were treated with rhein $(0,15,30,50 \mu \mathrm{M})$ for $24 \mathrm{hrs}$. Total protein was extracted, and the expression levels of P-JAK2, JAK and GAPDH proteins were measured by Western blot analysis. (F) HCTII 6 cells were treated with rhein $(0,15,30,50 \mu \mathrm{M})$ for 24 hrs. And cells were treated with IL-6 for 30 mins, the expression levels of P-STAT3, STAT3 and GAPDH proteins were measured by Western blot analysis. (G) Western blot analysis results from P-STAT3 were calculated. (H) The combination effect of rhein and erlotinib on cell viability $(* * * P<0.00 I$, $* * * * P<0.000 \mathrm{I})$.

Abbreviations: CRC, colorectal cancer; EGFR, epidermal growth factor receptor. 

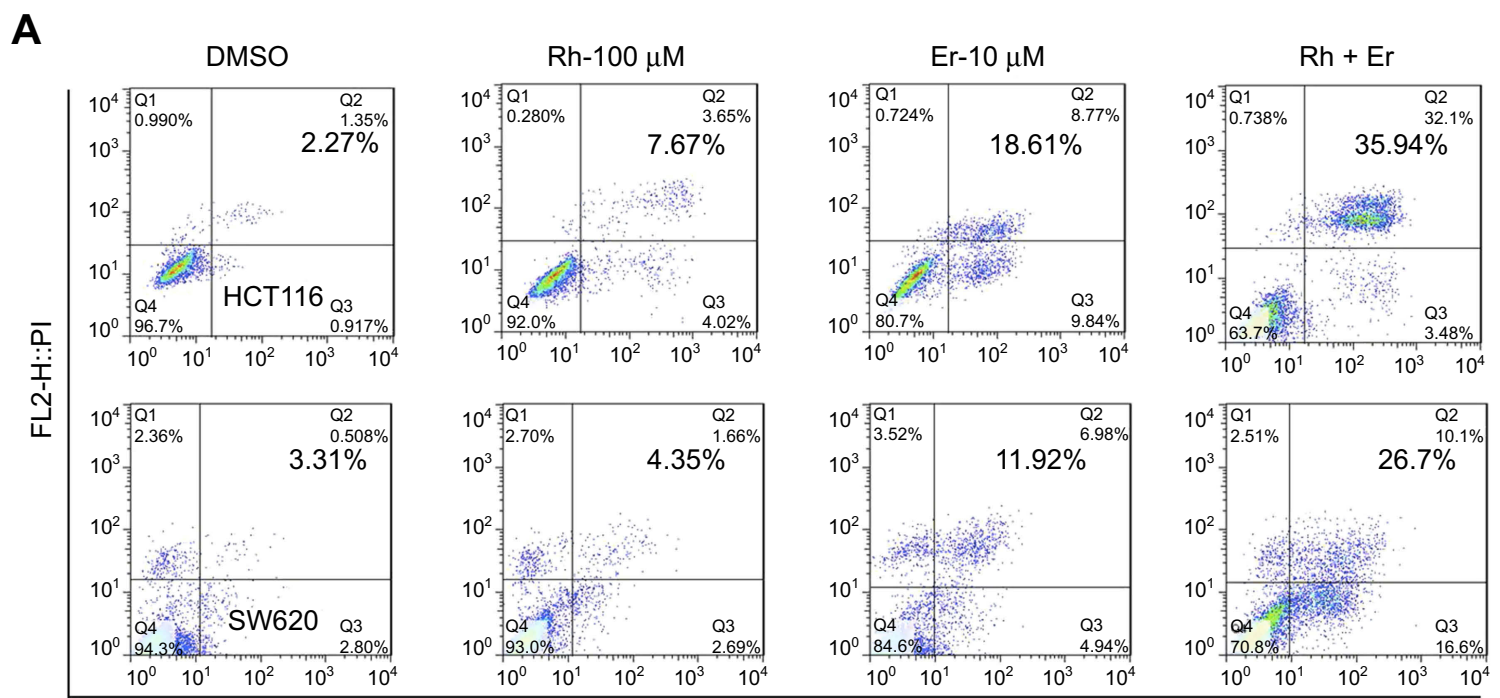

FL1-H::FITC-V

B
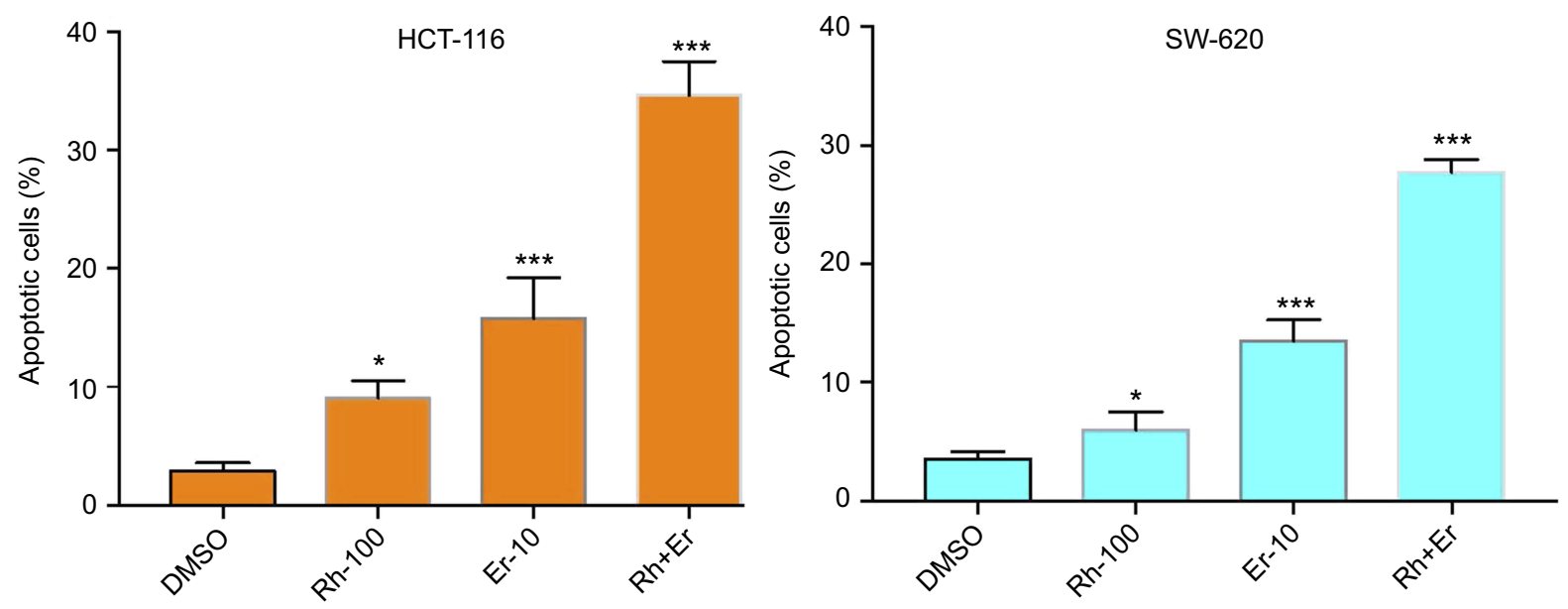

Figure 4 Induction of apoptosis in CRC cells by the combination of rhein and erlotinib. (A) HCTII6 and SW620 cells were treated, in combination or separately, with erlotinib $(\mathrm{Er} ; 10 \mu \mathrm{M})$ and/or rhein $(\mathrm{Rh} ; 100 \mu \mathrm{M})$ for $24 \mathrm{~h}$. The level of apoptosis was evaluated by analyzing Annexin $\mathrm{V}$ and PI staining, by flow cytometry analysis. (B) A statistical diagram of cell apoptosis $(* P<0.05$, $* * * P<0.00 \mathrm{I})$.

Abbreviation: CRC, colorectal cancer.

\section{The combination treatment with rhein and erlotinib induces cell cycle arrest at G2/M in human CRC cells}

The cell cycle process involves a series of cellular events that lead to cell division and eventually proliferation. To determine the effect of rhein and erlotinib on the cell cycle in human CRC cells, HCT116 and SW620 cells were treated with rhein $(100 \mu \mathrm{M})$, erlotinib $(10 \mu \mathrm{M})$, and rhein $(100 \mu \mathrm{M})+$ erlotinib $(10 \mu \mathrm{M})$ for $24 \mathrm{~h}$, and stained with Annexin V labeled with fluorescein isothiocyanate (Annexin V-FITC) and PI for flow cytometry analysis. Data analysis revealed that treatment with the combination of the two drugs resulted in a significant increase in the number of cells arrested at the $\mathrm{G} 2 / \mathrm{M}$ phase (Figure $5 \mathrm{~A}$ and $\mathrm{B}$ ).

\section{The combination of rhein and erlotinib synergistically inhibited cell colony formation} The changes in cells morphology reflect the anticancer effect of rhein and erlotinib alone or in combination. The most obvious cellular morphological changes caused by the treatment with the combination of the two drugs are shown in Figure 6A. We also evaluated the effect of rhein and erlotinib on the colony formation ability of HCT116 CRC cells. The results confirmed that the colony formation ability of cells in the group treated 
A
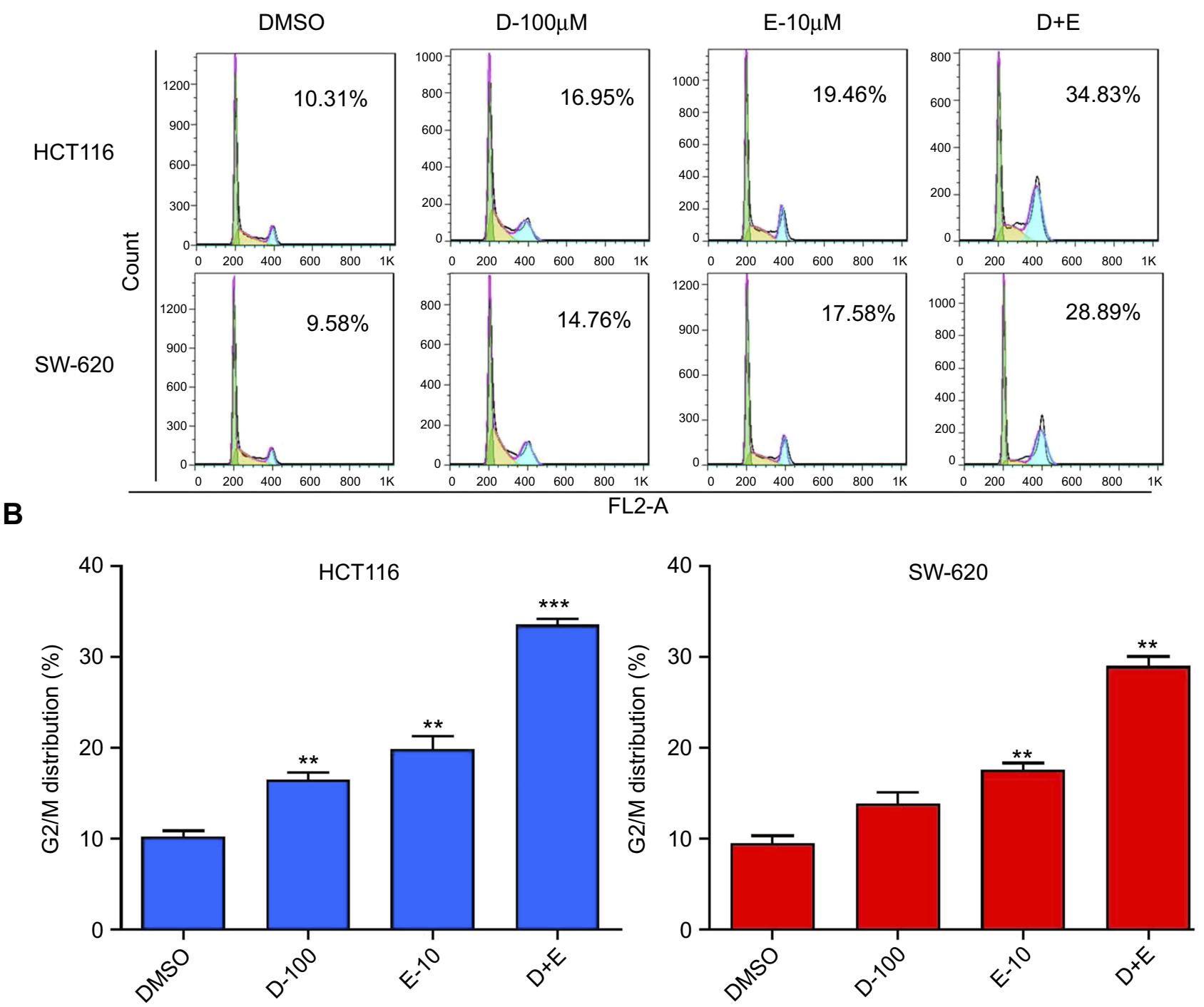

Figure 5 Rhein $(100 \mu \mathrm{M})$ and erlotinib (10 $\mu \mathrm{M})$, in combination or separately, cause cell cycle arrest in colon cancer cells. (A) Flow cytometry analysis of two different colon cancer cell lines, HCTII6 and SW620. (B) A statistical diagram of cell cycle arrest by flow cytometry analysis. $(* * P<0.01, * * * P<0.00 \mathrm{I})$.

with the combination of rhein and erlotinib was significantly inhibited compared with that of the cells in the group treated with a single drug (Figure 6B). Taken together, these data clearly demonstrated that the combination of rhein and erlotinib can effectively inhibit the proliferation of CRC cells.

\section{Combination treatment with rhein and erlotinib efficiently synergistically suppresses phosphorylation of STAT3 and EGFR in human CRC cell lines}

To study the mechanism of the synergistic effect of rhein and erlotinib, we analyzed HCT116 cells treated with a combination of erlotinib and rhein or with either drug alone, for $24 \mathrm{~h}$, by Western blot analysis. The results indicated that the combination treatment markedly decreased P-EGFR and P-STAT3 expression (Figure 6C, S1A and B). In addition, Western blot analysis further revealed that the combination treatment with erlotinib and rhein decreased the expression level of the anti-apoptotic protein BCL2 and cyclin-related proteins CDC2, Cyclin B1 and Cyclin D1 and increased the level of anti-apoptotic protein BAX in a dosedependent manner (Figure 6D, S1C-G).

\section{Discussion}

$\mathrm{CRC}$ is a malignancy with high morbidity and mortality, and is the second most common cause of cancer death in 
A
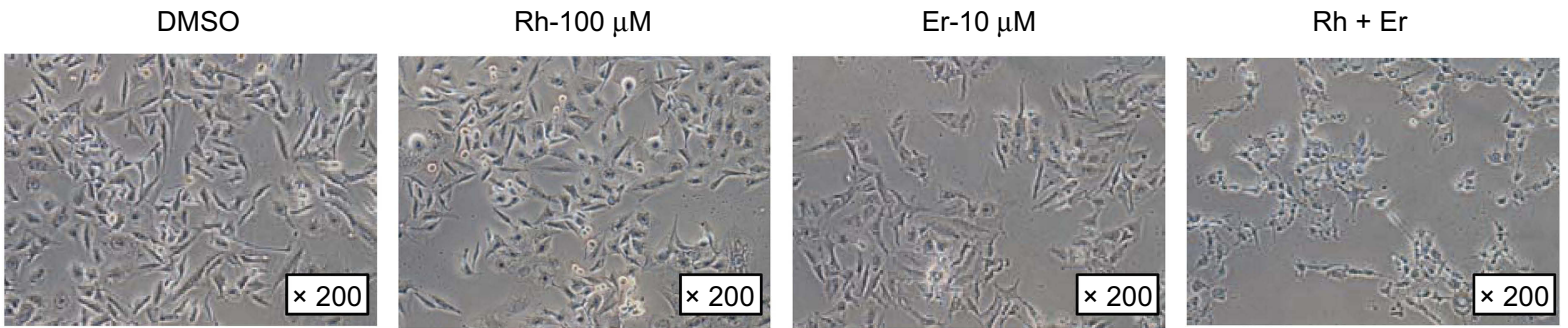

B

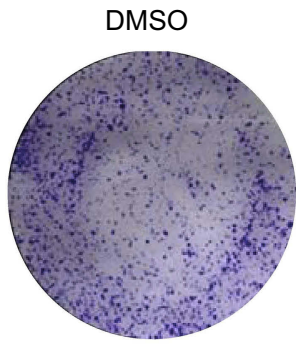

Rh-100 $\mu \mathrm{M}$

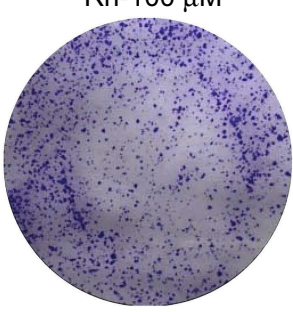

C

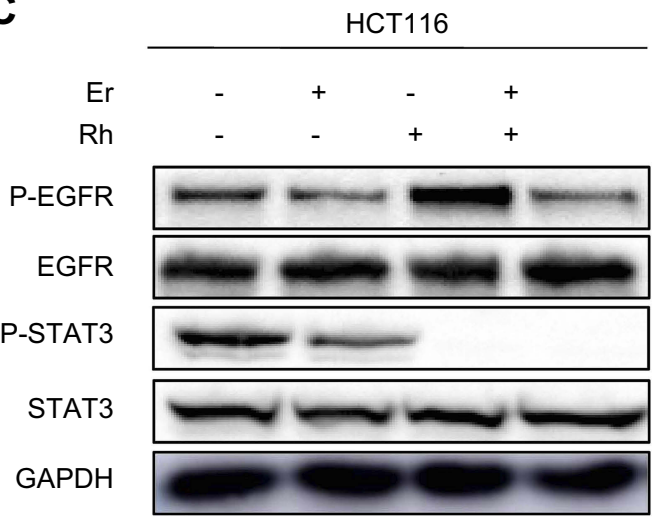

Er-10 $\mu \mathrm{M}$

$\mathrm{Rh}+\mathrm{Er}$
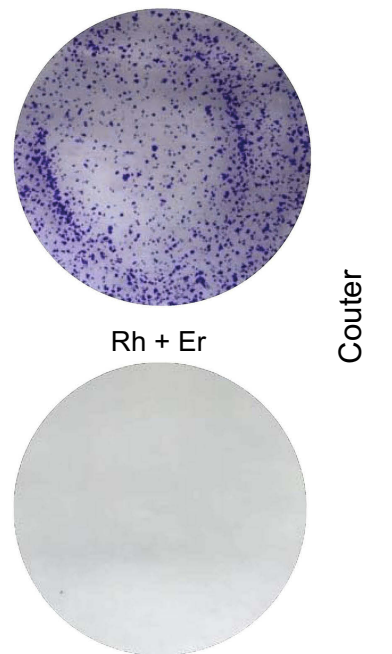

D
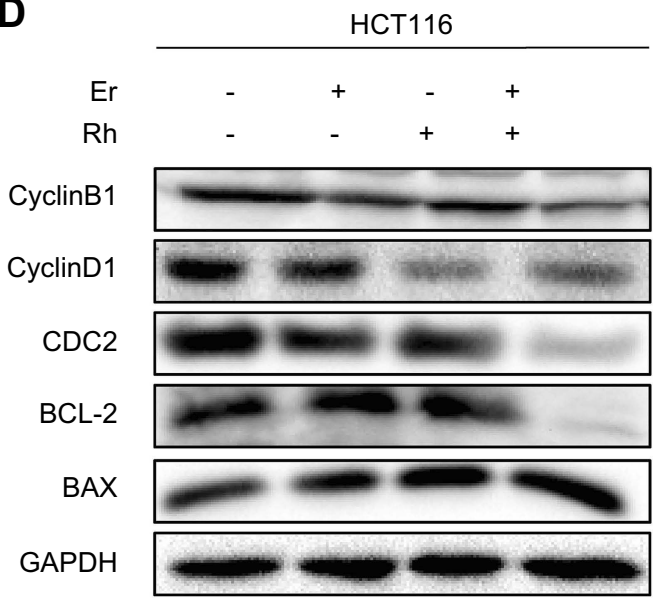

Figure 6 Rhein and erlotinib efficiently synergistically suppresses phosphorylation of STAT3 and EGFR. (A) Morphological changes of HCTII 6 colon cancer cells observed by light microscopy after drug treatment. (B) Colony formation assay of HCTII6 cells. (C) HCTII6 cells were treated, in combination or separately, with erlotinib (Er; $10 \mu \mathrm{M})$ and/or rhein $(\mathrm{Rh} ; 50 \mu \mathrm{M})$ for $24 \mathrm{~h}$. P-STAT3, P-EGFR, STAT3 and EGFR were detected by Western blot analysis. GAPDH was used as the control protein. (D) HCTII 6 cells were treated, in combination or separately, with erlotinib (I0 $\mu$ M) and/or rhein $(50 \mu \mathrm{M})$ for $24 \mathrm{~h}$. CDC2, CyclinBI, CyclinDI, BCL2 and BAX were detected by Western blot analysis, GAPDH was used as the control protein, $* * * * P<0.000 \mathrm{I}$.

human worldwide. ${ }^{2}$ The development of targeted drugs is an important breakthrough in the treatment of CRC, especially for targeting EGFR. ${ }^{5}$ However, with the popularity of targeted drugs, the emergence of drug resistance has become a difficult problem associated with the use of antitumor drugs. ${ }^{1,7}$ Further research on the drug resistance mechanism to find ways to overcome drug resistance, is an urgent problem to be solved in the field of CRC therapy.

STAT3 is an emerging target for cancer therapy. ${ }^{10}$ It is established that STAT3 mediates a variety of cellular functions, including proliferation, cell differentiation, metastasis, angiogenesis, apoptosis and immune response. ${ }^{13,19}$ 
Some reports show that STAT3 itself induces CRC. ${ }^{20}$ Most importantly, STAT3 has been formally identified as a molecular link between chronic inflammatory bowel disease and CRC. ${ }^{11}$ Numerous related studies have reported that the activation status of STAT3 has a great impact on the therapeutic effect of EGFR-TKIs. ${ }^{13}$ An increasing number of studies have shown that abnormal activation of STAT3 occurs in a variety of drug-resistant cells, and inhibition of STAT3 can reverse drug resistance. ${ }^{17}$ Therefore, STAT3 is deemed as an attractive target for antitumor drug development. ${ }^{21,22}$

In this study, we demonstrated that rhein, a new type of STAT3 inhibitor, sensitizes EGFR inhibitors to antitumor effect, further showing that STAT3 may be a direct target of rhein. Although we established that rhein and EGFR inhibitors have synergistic antitumor effect on CRC, the mechanism of action of rhein is not known in enough detail. Clinical trials with a combination of rhein and EGFR-TKIs are expected as a new therapeutic strategy.

\section{Conclusions}

Overall, we identified rhein as a new type of STAT3 small molecule inhibitors. Furthermore, our results suggested that rhein sensitizes human CRC cells to EGFR inhibitors possibly through down-regulating STAT3 signaling. Rhein, in combination with other EGFR targeting agents, could be further developed as a potential therapy for CRC.

\section{Acknowledgments}

This work was financially supported by the Zhejiang Provincial Natural Science Foundation of China (LY17H160055), Medical Scientific Research Fund of Zhejiang Province (2019322308 and 2017192276) and Wenzhou Science and Technology Project (Y20170280).

\section{Disclosure}

The authors report no conflicts of interest in this work.

\section{References}

1. Misale S, Yaeger R, Hobor S, et al. Emergence of KRAS mutations and acquired resistance to anti-EGFR therapy in colorectal cancer. Nature. 2012;486:532-536. doi:10.1038/nature11156

2. Siegel RL, Miller KD, Jemal A. Cancer statistics, 2019. CA Cancer J Clin. 2019;69:7-34.

3. Wolf AMD, Fontham ETH, Church TR, et al. Colorectal cancer screening for average-risk adults: 2018 guideline update from the American Cancer Society. CA Cancer J Clin. 2018;68:250-281.
4. Okada Y, Kimura T, Nakagawa T, et al. EGFR downregulation after Anti-EGFR therapy predicts the antitumor effect in colorectal cancer. Mol Cancer Re. 2017;15:1445-1454. doi:10.1158/1541-7786.MCR17-0157

5. Wu P, Nielsen TE, Clausen MH. FDA-approved small-molecule kinase inhibitors. Trends Pharmacol Sci. 2015;36:422-439. doi:10.1016/j.tips.2015.04.005

6. Bertotti A, Papp E, Jones S, et al. The genomic landscape of response to EGFR blockade in colorectal cancer. Nature. 2015;526:263-267. doi:10.1038/nature14969

7. Misale S, Di Nicolantonio F, Sartore-Bianchi A, Siena S, Bardelli A. Resistance to anti-EGFR therapy in colorectal cancer: from heterogeneity to convergent evolution. Cancer Discov. 2014;4:1269-1280. doi: 10.1158/2159-8290.CD-13-0646

8. Troiani T, Martinelli E, Napolitano S, et al. Increased TGF-alpha as a mechanism of acquired resistance to the Anti-EGFR inhibitor cetuximab through EGFR-MET interaction and activation of MET signaling in colon cancer cells. Clin Cancer Res. 2013;19:6751-6765. doi:10.1158/1078-0432.CCR-13-0423

9. Miroddi M, Sterrantino C, Simmonds M, et al. Systematic review and meta-analysis of the risk of severe and life-threatening thromboembolism in cancer patients receiving anti-EGFR monoclonal antibodies (cetuximab or panitumumab). Int $J$ Cancer. 2016;139:2370-2380. doi:10.1002/ijc.v139.10

10. Johnson DE, O'Keefe RA, Grandis JR. Targeting the IL-6/JAK/ STAT3 signalling axis in cancer. NAt Rev Clin Oncol. 2018;15:234-248. doi:10.1038/nrclinonc.2018.8

11. Huynh J, Chand A, Gough D, Ernst M. Therapeutically exploiting STAT3 activity in cancer - using tissue repair as a road map. Nat Rev Cancer. 2019;19:82-96. doi:10.1038/s41568-018-0090-8.

12. Yang L, Lin S, Kang Y, et al. Rhein sensitizes human pancreatic cancer cells to EGFR inhibitors by inhibiting STAT3 pathway. $J$ Exp Clin Cancer Res. 2019;38:31. doi:10.1186/s13046-018-1015-9

13. Zhao CG, Li HM, Lin HJ, Yang SL, Lin JY, Liang G. Feedback activation of STAT3 as a cancer drug-resistance mechanism. Trends Pharmacol Sci. 2016;37:47-61. doi:10.1016/j.tips.2015.10.001

14. Shi LY, Zheng HL, Hu WL, et al. Niclosamide inhibition of STAT3 synergizes with erlotinib in human colon cancer. Oncotargets Ther. 2017;10:1767-1776. doi:10.2147/OTT.S129449

15. Zhao CG, Xiao H, Wu XJ, et al. Rational combination of MEK inhibitor and the STAT3 pathway modulator for the therapy in K-Ras mutated pancreatic and colon cancer cells. Oncotarget. 2015;6:14472-14487.

16. Beebe JD, Liu JY, Zhang JT. Two decades of research in discovery of anticancer drugs targeting STAT3, how close are we? Pharmacol Ther. 2018;191:74-91. doi:10.1016/j.pharmthera.2018.06.006

17. Zheng H, Yang L, Kang Y, et al. Alantolactone sensitizes human pancreatic cancer cells to EGFR inhibitors through the inhibition of STAT3 signaling. Mol Carcinog. 2019;58:565-576. doi:10.1002/ mc.22951.

18. Yang LH, Li JF, Xu LY, et al. Rhein shows potent efficacy against non-small-cell lung cancer through inhibiting the STAT3 pathway. Cancer Manag Res. 2019;11:1167-1176. doi:10.2147/CMAR.S171517

19. Zheng HL, Hong H, Zhang LL, et al. Nifuratel, a novel STAT3 inhibitor with potent activity against human gastric cancer cells. Cancer Manag Res. 2017;9:565-571. doi:10.2147/CMAR. S146173

20. Wei J, Ma L, Lai YH, et al. Bazedoxifene as a novel GP130 inhibitor for Colon Cancer therapy. J Exp Clin Cancer Res. 2019;38:63. doi:10.1186/s13046-019-1072-8

21. Bai EC, Yang LH, Xiang YQ, et al. L61H46 shows potent efficacy against human pancreatic cancer through inhibiting STAT3 pathway. Cancer Manag Res. 2018;10:565-581. doi:10.2147/CMAR.S159090

22. Bin Z, Hong Z, Zheng HL, et al. Pectolinarigenin suppresses pancreatic cancer cell growth by inhibiting STAT3 signaling. Nat Prod Commun. 2017;12:1861-1864. 


\section{Supplementary material}

A

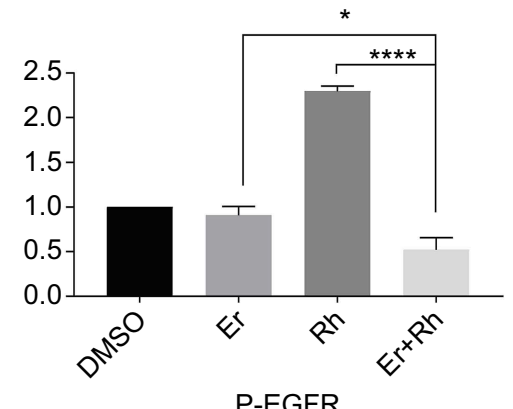

D

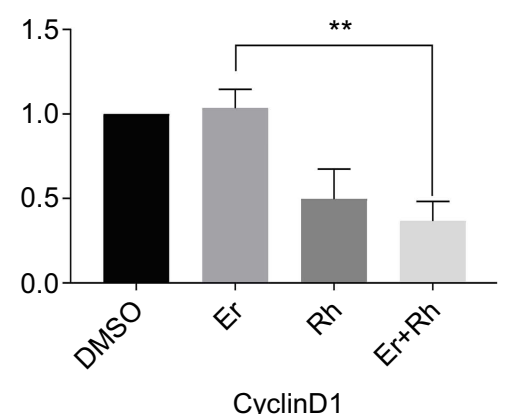

G

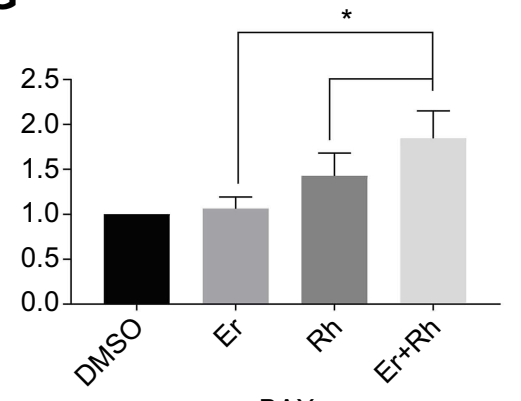

B

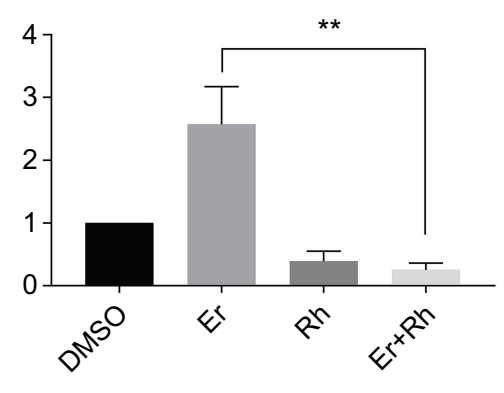

E

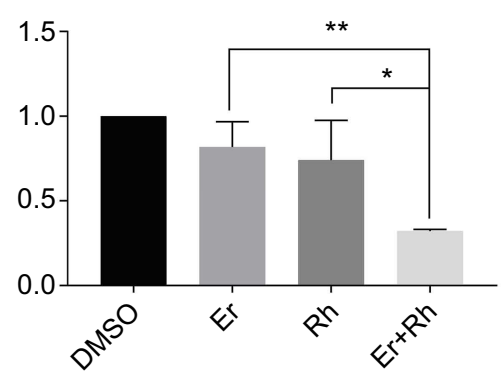

CDC2
C

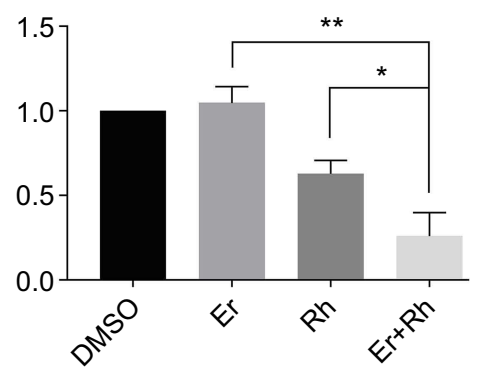

CyclinB1

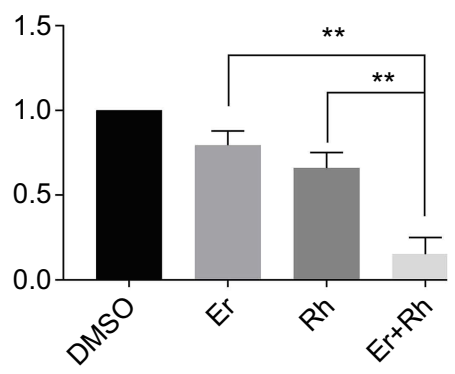

BCL-2

BAX

Figure SI Western blot analysis results were calculated. (A) Western blot analysis results from P-EGFR were calculated. (B) Western blot analysis results from P-STAT3 were calculated. (C) Western blot analysis results from CyclinBI were calculated. (D) Western blot analysis results from CyclinDI were calculated. (E) Western blot analysis results from CDC2 were calculated. (F) Western blot analysis results from BCL-2 were calculated. (G) Western blot analysis results from BAX were calculated. $(* P<0.05, * * P<0.01, * * * * P<0.0001)$.

Abbreviations: EGFR, epidermal growth factor receptor; Rh, rhein; Er, erlotinib.

\section{Publish your work in this journal}

OncoTargets and Therapy is an international, peer-reviewed, open access journal focusing on the pathological basis of all cancers, potential targets for therapy and treatment protocols employed to improve the management of cancer patients. The journal also focuses on the impact of management programs and new therapeutic agents and protocols on patient perspectives such as quality of life, adherence and satisfaction. The manuscript management system is completely online and includes a very quick and fair peer-review system, which is all easy to use. Visit http://www.dovepress.com/ testimonials.php to read real quotes from published authors. 